\title{
Zum Einfluß geburtshilflich-perinatologischer Maßnahmen auf die Mortalität und Frühmorbidität von Frühgeborenen der Gewichtsklasse 500 bis 1500 Gramm
}

\author{
H. J. Voigt, N. Lang, H. Segerer, K. Stehr* \\ Klinik für Frauenheilkunde mit Poliklinik und Hebammenschule der Universität Erlangen-Nürnberg \\ (Direktor: Prof. Dr. N. Lang) \\ Klinik mit Poliklinik für Kinder und Jugendliche der Friedrich-Alexander-Universität Erlangen-Nürnberg \\ (Direktor: Prof. Dr. K. Stehr)*
}

\section{Zusammenfassung}

In einer retrospektiven Analyse der perinatalen Einflußfaktoren von 186 Frühgeborenen der Erlanger Universitäts-Frauenklinik aus den Jahren 1982 bis 1987 mit einem Geburtsgewicht von 500 bis1500 g wurde die Mortalität und die Frühmorbidität, charkterisiert durch Hirnblutungen, Atmenotsyndrom und Infektionen in ihrem Zusammenhang mit dem geburtshilflichen Vorgehen und der pädiatrischen Intensivbehandlung untersucht. Es wurden 45 Kinder der Jahrgänge 1982/83 den 141 Kindern, die von 1984 bis 1987 einem veränderten Behandlungsprinzip unterworfen waren, gegenübergestellt. Im zweiten Untersuchungszeitraum ließ sich eine deutliche Senkung der Mortalität und der Inzidenz der asphyxie-bedingten, schweren Hirnblutungen und des Atemnotsyndroms beobachten. Als signifikante geburtshilfliche Einflußfaktoren erwiesen sich ein großzügigerer Entschluß zur operativen Entbindung durch die Sectio, die Verkürzung der Latenzzeit nach vorzeitigem Blasensprung und die stärkere Präsenz eines neonatologisch erfahrenen Teams von Pädiatern. Die beobachtete Verdreifachung der Sectiofrequenz hat zu einer Reduktion der perinatalen Mortalität und Morbidität um $50 \%$ geführt. Von der großzügigen Indikation zur Sectio profitieren vor allem die BEL-Kinder, bedingt auch die Schädellagen-Kinder. Die Verkürzung der Latenzphase bei vorzeitigem Blasensprung führte zu einer deutlichen Senkung der Infektionsmorbidität und Mortalität. Es wird daraus der Schluß gezogen, daß das häufig geübte abwartende Verhalten mit dem Ziel, die Lungenreife zu verbessern, zugunsten einer aktiveren, die Infektion und das Geburtstrauma vermeidenden Geburtsleitung aufgegeben werden sollte. Die Basis für geburtshilfliche Entscheidungen sollte mehr die pränatale Gewichtsschätzung als das errechnete Gestationsalter sein. Als untere Grenze des mit einem gesunden Leben zu vereinbarenden Geburtsgewichtes werden derzeit $750 \mathrm{~g}$ angesehen.

\author{
Influence of Obstetric-Perinatological \\ Measures on Mortality and Early \\ Morbidity of Premature Newborn Children \\ Weighing 500-1500 Grammes
}

In a retrospective analysis of perinatal influencing factors in 186 premature newborns of the Department of Gynaecology of the University of Erlangen covering the period from 1982-1987 with birth weights between 500 and 1500 grammes, the mortality and early morbidity were analysed, as characterised by cerebral haemorrhages, respiratory distress syndrome and infections insofar, as they had been connected with the obstetrical approach and paediatric intensive-care treatment. 45 infants born in 1982/ 83 were compared with 141 infants, who had been subjected to a different treatment approach during 1984 to 1987 . During the second period, there was a marked drop both in mortality and in the incidence of asphyxia-induced severe cerebral haemorrhage and of the respiratory distress syndrome. A shortened latency period after premature rupture of the amnion, and a more pronounced presence of a neonatologically experienced team of paediatricians were found to be significant obstetric liberal influencing factors in determining the need to perform Caesarian section. The triplication of the frequency of Caesarean section observed resulted in a $50 \%$ reduction in perinatal mortality and morbidity. Infants with pelvic presentation benefited most from the more liberal performance of Caesarean section, as did infants with vertex presentation. Shortening of the latency phase in premature rupture resulted in a marked reduction in infection morbidity and mortality. Therefore we conclude, that the frequently practised procrastination with the aim to await an improvement in lung maturity should be replaced by a more active obstetric management, avoiding both infection and birth trauma. Obstetric decisions should be based rather on prenatal estimation of weight than on the calculated gestational age. At present, the lowest birth weight associated with the expectation of a healthy life is considered to be 750 grammes.

Herrn Dr. W. Grünsteidel, Universitäts-Frauenklinik, danken wir für die statistischen Berechnungen. 


\section{Einleitung}

Die Überlebenschancen für Früh- und Frühmangelgeborene konnten in den letzten Jahren entscheidend verbessert werden $(7,9,14)$. Dies ist sicher in erster Linie als ein Erfolg der neonatalen Intensivmedizin zu werten. Eine entscheidende Voraussetzung hierfür stellt jedoch der „Zustand“ dar, in dem die unreifen und untergewichtigen Kinder dem Neonatologen übergeben werden. Damit stehen Fragen nach dem geburtshilflichen Management, der Regionalisierung von Frühgeburten sowie der Zusammenarbeit zwischen Geburtshelfern und Pädiatern in einem perinatalmedizinischen Zentrum im Vordergrund.

In einer ersten Analyse des neonatologischen Schicksals kleinster Früh- und Frühmangelgeborener der Erlanger Universitäts-Frauenklinik mit einem Geburtsgewicht von weniger als $1500 \mathrm{~g}$ haben u. a. Segerer et al. (23) gezeigt, daß die Anwesenheit eines pädiatrischen Intensivmedizinerteams die engste Beziehung zum günstigen Verlauf dieser Frühgeburten hatte. $\mathrm{Zu}$ ähnlichen Resultaten kamen auch andere Autoren $(16,21)$.

Es sollte in dieser Untersuchung der Frage nachgegangen werden, welche speziellen geburtshilflichen Bedingungen möglicherweise das neonatologische Ergebnis beeinflußt haben. Hierzu wurde der Einfluß der Kortikoid-Prophylaxe, der Latenzzeit zwischen vorzeitigem Blasensprung und der Geburt und schließlich der Frequenz der Entbindung durch die Sectio in einer retrospektiven Analyse von 186 Geburten sehr kleiner Frühgeborener $(<1500 \mathrm{~g})$ der Erlanger Universitäts-Frauenklinik aus den letzten 6 Jahren untersucht. Da sich im Jahre 1984 ein Wandel im geburtshilflichen Vorgehen der Klinik vollzog, bot sich auch ein historischer Vergleich durch Gegenüberstellung der Jahrgänge 1982/ 83 und 1984 bis 1987 an, während die neonatologische intensivmedizinische Abteilung im gesamten Zeitraum unter vergleichbaren apparativen und personellen Bedingungen tätig war. Geändert hatte sich lediglich die intensive Einbeziehurg der Neonatologie in die Geburtshilfe der Universitäts-Frauenklinik in Form einer pädiatrischen Dauerpräsenz während der Tagesdienstzeit.

\section{Patienten und Methoden}

In einer retrospektiven Analyse wurden 186 Kinder erfaßt, die bei 153 Entbindungen an der Universitäts-Frauenklinik Erlangen zwischen Januar 1982 und Dezember 1987 geboren wurden. Die Geburtsgewichte lagen zwischen 500 und $1500 \mathrm{~g}$ (WHO-Definition: very low birth weight). Es wurden nach AusschluB der Totgeburten und der Kinder mit schicksalsbestimmenden, nicht mit dem Überleben zu vereinbarenden Fehlbildungen $(n=52)$ alle Frühgeburten dieses Zeitraums erfaßt.

Um Veränderungen und Ergebnisse des geburtshilflichen und neonatologischen Vorgehens im zeitlichen Verlauf erfassen zu können, wurden die Kinder der Jahre 1982 und 1983 denen der Jahrgänge 1984 bis 1987 gegenübergestellt. Das sonographische Schätzgewicht war im gesamten Zeitraum nach den Tabellen von llansmann et al. (6) ermittelt worden. Der Schweregrad der Hirnblutungen wurde sonographisch nach den anerkannten Kriterien von Papile et al. (17) oder nach dem Obduktionsbefund eingeteilt. Die Einteilung des Atemnotsyndroms erfolgte nach den röntgenologischen Kriterien von Giedion et al. (5) in vier Schweregrade.

Der Einfluß geburtshilflicher Maßnahmen wie Vollständigkeit der Kortikoid-Prophylaxe. Frequenz der operativen Entbin- dung sowie Latenzzeit zwischen vorzeitigem Blasensprung und Geburt auf das Auftreten kindlicher Hirnblutungen, neonataler Infektionen, das Atemnotsyndrom sowie auf die perinatale kindliche Mortalität wurden geprüft.

Bei der statistischen Auswertung wurde für den Vergleich von Häufigkeiten der Chi-Quadrat-Test, bei kleiner Fallzahl der Fisher-Test eingesetzt. Nicht normal verteilte Daten wie Geburtsgewichte und Schwangerschaftsdauer wurden mit dem U-Test nach Mann-Whitney verglichen.

\section{Ergebnisse \\ Geburtshilfliche Basisdaten}

Um die Vergleichbarkeit der Kollektive zu prüfen, wurden die Mittelwerte und Standardabweichungen der Geburtsgewichte und der durchschnittlichen Schwangerschaftsdauer der 45 in den Jahren 1982/83 geborenen Kindern den Kindern der Jahre 1984 bis 1987 gegenübergestellt. Signifikante Unterschiede konnten nicht festgestellt werden (Tab. 1). Der Häufigkeitsvergleich der erkannten Ursachen, die zur Frühgeburt führten, wie der vorzeitige Blasensprung, vorzeitige Wehen, Blutungen infolge Plazenta praevia, die vorzeitige Plazentalösung, die Präeklampsie und die chronische Plazentainsuffizienz ergab für beide Zeiträume keinen signifikanten Unterschied.

Der Anteil der mangelgeborenen Kinder (SGA) scheint mit $24.4 \%$ in den Jahren $1982 / 83$ gegenüber $35,4 \%$ der Jahre 1984 bis 1987 geringer. Läßt man bezogen auf die Hohenauer-Kurve (10) die Kinder im Unsicherheitsbereich unterhalb der 28. SSW sowie die Mehrlinge unberücksichtigt. so findet sich kein signifikanter Unterschied mehr zwischen $17,8 \%$ von $1982 / 83$ und $24,8 \%$ von 184 bis 1987 . Der Anteil der Mehrlinge in den beiden Untersuchungszeiträumen war nicht signifikant verschieden. Das Gestationsalter betrug im Durchschnitt 29 Schwangerschaftswochen.

Das für die geburtshilfliche Entscheidung wichtige, sonographisch ermittelte Schätzgewicht zeigte in beiden Gruppen eine bemerkenswert gute Übereinstimmung mit dem tatsächlichen Geburtsgewicht. Somit kann von vergleichbaren Kollektiven ausgegangen werden.

Bei annähernd gleicher jährlicher Geburtenzahl in den Jahren 1982 bis 1987 (insgesamt 11963 Entbindungen) stieg der Anteil der für diese Untersuchung relevanten Kinder von $1 \%$ in den Jahren $1982-83$ auf $2,4 \%$ im Jahre 1987 stetig an.

\section{Einflußgeburtshilflicher Maßnahmen im zeitlichen Vergleich}

Die Gabe von Betametason zur Induktion der fetalen Lungenreifung erfolgte in beiden untersuchten Zeiträumen gleich häufig. Die Kortikoid-Prophylaxe wurde nur dann als ausreichend angesehen, wenn zwischen der Verabreichung und der Entbindung mindestens 48 Stunden lagen. Um dies zu erreichen, mußte in beiden Kollektiven annähernd gleich oft eine intravenöse, tokolytische Therapie vorgenommen werden. Die Gegenüberstellung der perinatalen Bedingungen zeigt Tabelle 2. 


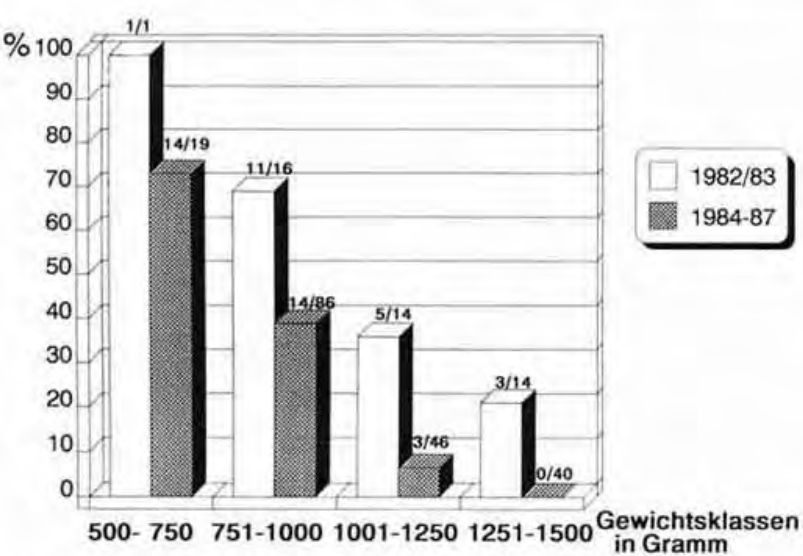

Abb. 1 Mortalität der Gewichtskiassen 1982/83 gegenüber 1984-87.

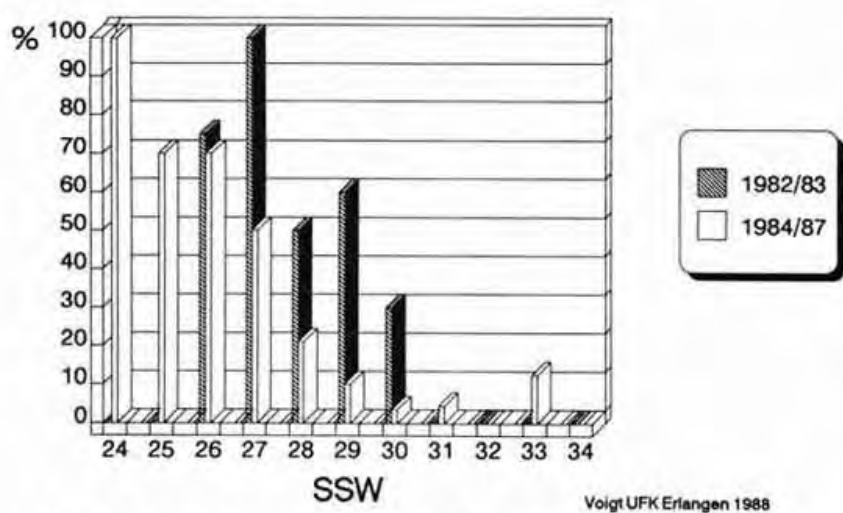

Abb. 2 Mortalität in den Schwangerschaftswochen.

\section{Abhängigkeit der kindlichen Morbidität und Mortalität von den perinatalen Faktoren}

Der Vergleich zweier Zeitabschnitte erlaubt es noch nicht, die Bedeutung der einzelnen geburtshilflichen Maßnahmen für die erzielte Verbesserung der geburtshilflichen Ergebnisse zu beurteilen. Wir haben daher die direkte Abhängigkeit der kindlichen Morbidität und Mortalität von meßbaren geburtshilflichen Einflußfaktoren geprüft.

\section{Kortikoid-Prophylaxe}

Ein schweres Atemnotsyndrom (Grad III und IV) trat in der Gruppe der Kinder mit einem Gewicht von 500 bis $1000 \mathrm{~g}$ ohne Kortikoid-Prophylaxe fast dreimal so häufig auf $(36 \%$ versus $13 \%$ ) wie bei Kindern, bei denen mindestens 48 Stunden vor der Entbindung Betametason in einer Dosierung von $2 \times 12 \mathrm{mg}$ im Abstand von 24 Stunden verabreicht worden war. Die Mortalität war vierfach höher $(48 \%$ versus $12,5 \%$ ). In der Gewichtsklasse 1001 bis $1500 \mathrm{~g}$ war dieser Effekt nicht mehr zu beobachten. Die Kinder ohne oder mit leichtgradigem ANS zeigten keinen Unterschied, auch nicht

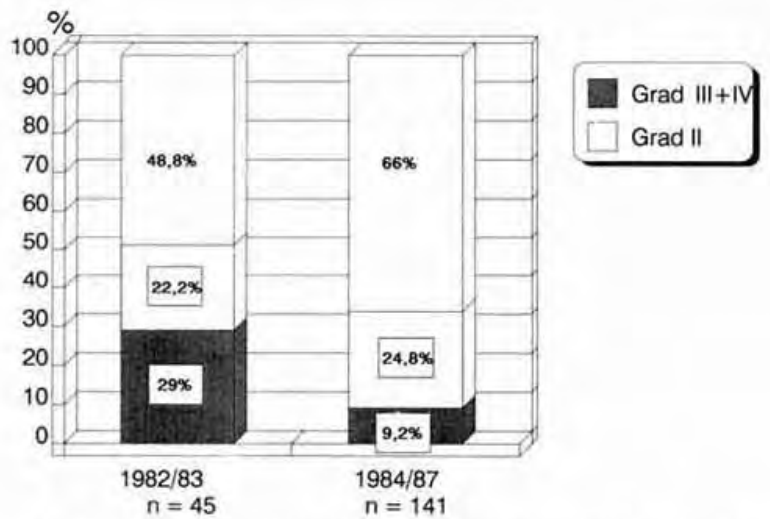

Abb. 3 Vorkommen von Hirnblutungen zweiten bis vierten Grades (Frühgeborene $500-1500 \mathrm{~g}$ ). Für die Häufigkeitsabnahme der dritt- und viertgradigen Blutungen: $p \ll 0.01$

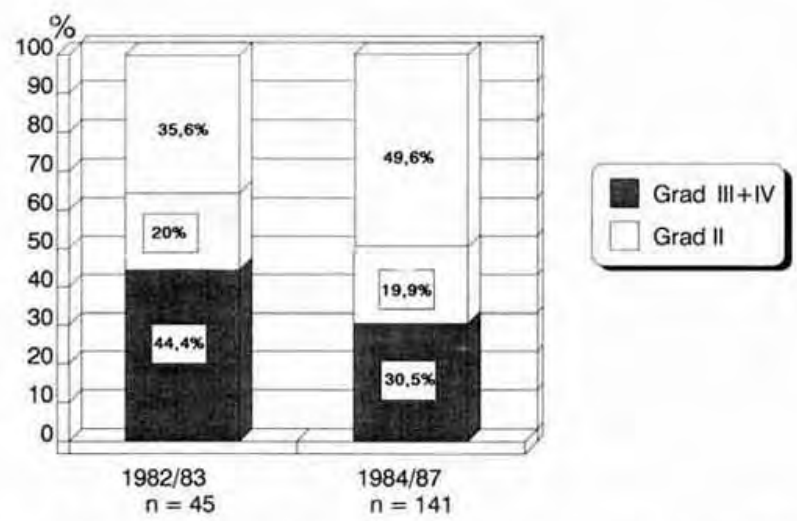

Abb. 4 Vorkommen des Atemnotsyndroms zweiten bis vierten Grades (Frühgeborene $500-1500 \mathrm{~g}$ ). Die Häufigkeitsabnahme des dritt-und viertgradigen Atemnotsyndroms war nicht signifikant.

unter Berücksichtigung der Gewichtsklasse. Demnach profitieren nur Kinder mit einem Gewicht von 500 bis 1000 g nachweisbar von der Kortikoid-Prophylaxe. Die Kortikoid-Gabe hatte keinen Einfluß auf das Auftreten kindlicher perinataler Infektionen. Diese wurden in der Gruppe mit und ohne Prophylaxe gleich häufig beobachtet ( 13 und $11 \%$ ).

Die Prüfung des Effekts der Tokolysedauer ist eng verknüpft mit der „Wirkdauer“ der Kortikoid-Prophylaxe. Beim Auftreten vorzeitiger Wehen mit oder ohne Blasensprung wurde nach Ausschluß eines infektiösen Geschehens stets mit dem Ziel tokolysiert, den Geburtstermin hinauszuschieben, bis die Kortikoid-Prophylaxe abgeschlossen werden konnte (in der Regel 48 Stunden).

\section{Latenzzeit zwischen Blasensprung und Geburt}

Ein vorzeitiger Blasensprung wurde $60 \mathrm{mal}$ beobachtet. $16 \mathrm{mal}$ kam es dabei zu einer kindlichen Sepsis. $13 \mathrm{mal}$ lag hierbei der Blasensprung mehr als 48 Stunden 
Tab. 4 Schweres ANS, schwere HB und Mortalität in Abhängigkeit vom Geburtsmodus in den Gewichtsklassen 500-1000 Gramm und 1001-1500 Gramm.

\begin{tabular}{|c|c|c|c|c|c|c|c|c|c|c|}
\hline $500-1000$ Gramm & ANS & & & $\mathrm{HB}$ & & & $A N S+H B$ & 1 & Mortalitāt & \\
\hline $\begin{array}{l}\text { Sectio } \\
\text { Spontangeburt } \\
\text { spontan Beckenendlage } \\
\text { spontan Schädellage }\end{array}$ & $\left.\begin{array}{rr}45,1 \% \\
65 & \% \\
75 & \% \\
60,7 \%\end{array}\right]$ & $\begin{array}{l}\text { n.s. } \\
\text { n.s. }\end{array}$ & n.s. & $\left.\begin{array}{l}12,9 \% \\
57,5 \% \\
83,3 \% \\
46,4 \%\end{array}\right]$ & $\begin{array}{l}p \ll 0,01 \\
p \ll 0,01\end{array}$ & $p \ll 0.01$ & 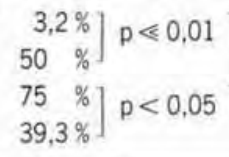 & $p \ll 0,01$ & 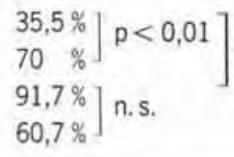 & ]$p<0,01$ \\
\hline $1001-1500$ Gramm & ANS & & & $\mathrm{HB}$ & & & $\mathrm{ANS}+\mathrm{HB}$ & & Mortalität & \\
\hline $\begin{array}{l}\text { Sectio } \\
\text { Spontangeburt } \\
\text { spontan Beckenendlage } \\
\text { spontan Schädellage }\end{array}$ & $\left.\begin{array}{l}17,1 \% \\
15,6 \% \\
25 \% \\
17,1 \%\end{array}\right]$ & n.s. & n.s. & $\left.\begin{array}{r}2,8 \% \\
11,1 \% \\
25 \% \\
12,3 \%\end{array}\right]$ & $\begin{array}{l}\text { n.s. } \\
\text { n.s. }\end{array}$ & $p<0,05$ & 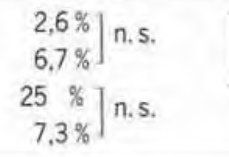 & $<0,05$ & 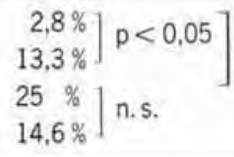 & $p<0,05$ \\
\hline
\end{tabular}

zurück. 8 Kinder starben ohne gleichzeitiges Vorliegen einer schweren Hirnblutung oder eines schweren ANS. Soweit es die kleinen Zahlen erlauben, läßt sich eine klare Abhängigkeit zwischen dem über 48 Stunden zurückliegenden Blasensprung und einer neonatalen Infektion sowie der Schwere des Verlaufs erkennen. Die Mortalität nahm mit zunehmendem Geburtsgewicht $\mathrm{ab}$

1982/83 betrug die mittlere Latenzzeit bei den durch Infektionen komplizierten Fällen mit Blasensprung 6 Tage und 5 Stunden und zeigte eine Sepsis-Rate von $39 \%$. Diese wurde 1984/87 auf $21 \%$ gesenkt. Hier betrug das Intervall im Mittel 2 Tage und 21 Stunden.

\section{Entbindungsmodus}

100 Kinder wurden durch Sectio geboren, 86 auf vaginalem Wege, davon 68 aus Schädellage und 18 aus Beckenendlage. Das Auftreten eines schweren Atemnotsyndroms oder einer schweren Hirnblutung sowie die Mortalität im Zusammenhang mit dem Geburtsmodus zeigt Tabelle 4. Mit Ausnahme der Häufigkeit des schweren ANS unterschieden sich spontan Geborene von den durch Kaiserschnitt entwickelten Kindern im Auftreten von schweren Hirnblutungen und in der perinatalen Mortalität. Am deutlichsten traten die schlechten Ergebnisse der Kinder unter 1000 g hervor, die aus Bekkenendlage auf vaginalem Wege geboren wurden. Die Häufigkeit der schweren Hirnblutungen $(83,3 \%)$ und die Mortalität $(91,7 \%)$ war hier mit Abstand am höchsten. Weniger deutlich waren die Unterschiede in der Gewichtsklasse 1001 bis

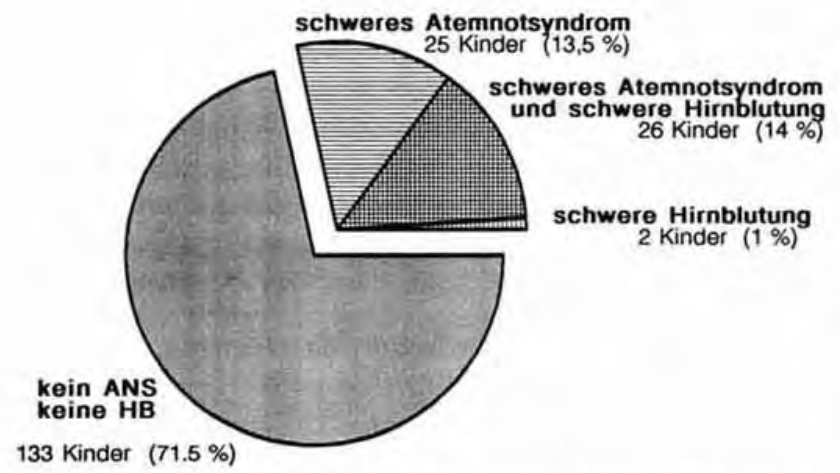

Abb. 5 Zusammentreffen von schwerem Atemnotsyndrom und schwerer Hirnblutung (Frühgeborene $750-1500$ g, 186 Kinder).
1500 g. Der Vergleich der durch Sectio geborenen BEL-Kinder mit den spontanen BEL-Geburten zeigte jedoch auch in dieser Gewichtsklasse signifikant bessere Resultate für die Schnittentbindung bezogen auf die Häufigkeit der schweren Hirnblutungen und die perinatale Mortalität.

Zwischen dem Auftreten eines Atemnotsyndroms und einer Hirnblutung fand sich eine hochsignifikante Beziehung (Abb. 5). Bei 71,5\% $(\mathrm{n}=133)$ der Frühgeborenen wurde weder ein schweres Atemnotsyndrom noch eine schwere Hirnblutung gefunden. 26 von 51 Kindern mit einem schweren Atemnotsyndrom erlitten ebenfalls eine schwere Hirnblutung. Bei den 135 Kindern ohne schweres Atemnotsyndrom wurde nur zweimal eine schwere Hirnblutung diagnostiziert. Die Gruppe der Kinder mit schweren Hirnblutungen und/oder schwerem Atemnotsyndrom $(\mathrm{n}=53)$ wies 36 Todesfälle auf $(68 \%)$. Das sind $2 / 3$ der Gesamtmortalität. Hier sind wiederum die Kinder zweifach überrepräsentiert, die vaginal aus Beckenlage entwickelt wurden. Ganz besonders sind Kinder unter $1000 \mathrm{~g}$ betroffen.

7 von 64 Kindern starben nach Sectio infolge einer repiratorischen Insuffizienz ohne gleichzeitige Hirnblutung. Von den 133 Kindern ohne schweres ANS und schwere HB starben $17(12,8 \%)$, die meisten $(\mathrm{n}=10)$ an einer perinatal erworbenen Infektion und Sepsis.

\section{Diskussion}

Vor 1960 war die Öberlebenschance für die kleinen Frühgeborenen der Gewichtsklasse 500 bis $1500 \mathrm{~g}$ sehr gering. Überleben bedeutete häufig auch ein Leben mit Behinderungen. In 40 bis $70 \%$ war mit geistigen und neurologischen Folgeschäden zu rechnen (9). Im letzten Jahrzehnt hat sich jedoch das Verhältnis von toten oder geschädigten Kindern zu den gesunden Überlebenden umgekehrt. Dank einer hochspezialisierten neonatalen Intensivmedizin konnte der Anteil der überlebenden Kinder stark verbessert und gleichzeitig der Anteil der behinderten Kinder gesenkt werden (22). Daher waren Geburtshelfer und Neonatologen gleichermaßen ermutigt, sich aktiver um diese kleinen Frühgeborenen zu kümmern, wo früher eher der natürliche Ablauf der Geburt abgewartet wurde und maternale Aspekte das geburtshilfliche Handeln stark beeinflußten (9).

Regionale Vergleichsstudien zeigten, wie beispielsweise die am University College Hospital London durch- 
Tab. 1 Basisdaten der verglichenen Jahrgänge.

\begin{tabular}{llll}
\hline & $1982-1983$ & $1984-1987$ & \\
\hline Geburtsgewicht & $1123+211^{\mathrm{a}}$ & $1076+261$ & n.s. $^{\text {b }}$ \\
sonogr. Schätzgewicht & $1101+151$ & $1097+135$ & n.s. \\
Schwangerschaftsdauer & $29,4+2,1$ & $29,5+2,4$ & n.s. \\
Anteil der SGA.Kinder & $24,4 \%$ & $35,4 \%$ & n.s. \\
$\begin{array}{l}\text { Anteil der SGA-Kinder } \\
\quad(>28 . \text { SSW, ohne Mehrlinge) }\end{array}$ & $17,8 \%$ & $24,8 \%$ & n.s. \\
$\begin{array}{l}\text { Mehrlinge } \\
\text { Menge }\end{array}$ & $28,8 \%$ & $33,3 \%$ & n.s. \\
\hline
\end{tabular}

a) Mittelwert \pm Standardabweichung; b) nicht signifikant

Tab. 2 Perinatale Bedingungen der kleinen Frühgeborenen (500 - $1500 \mathrm{~g}$ ) in Erlangen.

\begin{tabular}{l|l|l|l}
\hline & $1982-1983$ & $1984-1987$ & sign. \\
\hline Kortikoid.Prophylaxe & $51 \%$ & $51 \%$ & n.s. \\
i. v. Tokolyse & $57 \%$ & $51 \%$ & n.s. \\
vorz. Blasensprung & $38 \%$ & $20 \%$ & $p \ll 0,01$ \\
$\quad>48$ Std. & $24 \%$ & $63 \%$ & $p \ll 0,01$ \\
$\begin{array}{l}\text { Sectiorate } \\
\text { BEL spontan }\end{array}$ & $15,6 \%$ & $7,8 \%$ & $\mathrm{p}<0,05$ \\
$\quad$ neonatologische & $73 \%$ & $96 \%$ & $\mathrm{p} \ll 0,01$ \\
$\quad$ Erstversorgung & & & \\
$\quad \begin{array}{l}\text { Unterkühlung } \\
\quad<36^{\circ} \text { Crektal) }\end{array}$ & $45 \%$ & $54 \%$ & n.s. \\
\hline
\end{tabular}

Tab. 3 Geburtshilfliche Ergebnisse.

\begin{tabular}{l|c|c|c}
\hline & $1982-1983$ & $1984-1987$ & sign. \\
\hline $\begin{array}{l}\text { Asphyxie (Apgar 5 }<7 \\
\text { und/oder NA pH }<7,2)\end{array}$ & $44 \%$ & $36 \%$ & n. s. \\
$\begin{array}{l}\text { neonatale Mortalität } \\
\text { früh (pp bis 7 T.) }\end{array}$ & $28,9 \%$ & $15,6 \%$ & $\mathrm{p}<0,05$ \\
spät (pp bis $1 / 2 \mathrm{~J})$. & $46,7 \%$ & $22 \%$ & $\mathrm{p}<0,01$ \\
\hline $\begin{array}{l}\text { Anteil der Frühgeborenen } \\
\quad(500-1500 \text { g) an der }\end{array}$ & $1 \%$ & $2,4 \%$ & \\
$\quad \begin{array}{l}\text { ges. Kinderzahl } \\
\text { Anteil an der } \\
\text { Gesamt-Mortalität }\end{array}$ & $30 \%$ & $18 \%$ & \\
\end{tabular}

In den Jahren 1984 bis 1987 nahm die Zahl der Entbindungen mit über 48 Stunden zurückliegendem Blasensprung von $38 \%$ auf $20 \%$ deutlich ab (p $\ll 0,01$ ), da der Entschluß der vorzeitigen Entbindung häufiger und rascher gefaßt wurde. Läßt man die Fälle mit vorzeitigem Blasensprung vor der 27.SSW unberücksichtigt, wo abwartendes Verhalten verständlich ist. so stehen von 1982/83 35\% um so deutlicher gegenüber $13 \%$ im jüngeren Kollektiv. Bei Hinweisen auf ein beginnendes Amnion-Infektionssyndrom wie Temperatur >37 Grad Celsius, Leukozytenanstieg, fetale Tachykardie und Anstieg des CRP - seit 1986 eingesetzt - wurde stets unter antibiotischer Therapie umgehend entbunden.

Auffallend und statistisch hochsignifikant war bei den kleinen Frühgeborenen die Zunahme der Sectiorate von 24 auf $63 \%$ im zweiten Zeitraum. Dagegen halbierte sich der Anteil der Kinder, die aus Beckenendlage vaginal entwikkelt wurden, von 15,6 auf $7,8 \%$. Aus Beckenendlage wurden in den letzten beiden Jahren nur noch extreme Frühgeburten vor der 26. SSW vaginal geboren, deren Prognose als infaust eingestuft worden war.

In den Jahren 1984 bis 1987 war fast ohne Ausnahme ein pädiatrisches Team der neonatologischen Intensivstation zur Primärversorgung zugegen. Der Unterschied zur vorangehenden Periode ist signifikant. In beiden Kollektiven wurde etwa bei jedem zweiten Kind eine Unterkühlung nach Primärversorgung und Verlegung auf die pädiatrische Intensivstation festgestellt (Tab. 2).

Die geburtshilflichen Ergebnisse, gemessen an perinataler Mortalität und Frühmorbidität sind in Tabelle 3 aufgeführt. Bei der Asphyxierate zeigte sich eine Tendenz zur Abnahme, ohne jedoch statistische Beweiskraft zu erlangen.

Die neonatale Mortalität wurde als frühe neonatale Mortalität (bis 7 . Tag post partum) und späte Mortalität (pp. bis $1 / 2$ Jahr) getrennt analysiert, da Frühgeborene zum Teil auch nach einer Langzeitbeatmung und den Folgen der bronchiopulmonalen Dysplasie verstorben waren. In beiden Fällen zeigte sich gleichermaßen ein Rückgang auf die Hälfte.

Bezogen auf vier gebildete Gewichtsklassen fiel die Senkung der Mortalität unterschiedlich aus (Abb.1). Während 1982/83 kein Kind unter $750 \mathrm{~g}$ (Gewichtsklasse I) überlebte, gelang dies ab 1984 in 1/4 der Fälle (5 von 19. $=27 \%$ ). In der Gewichtsklasse III (1001 bis $1250 \mathrm{~g})$ wurden 1984 bis 1987 noch 3 von 46 Kindern $(=6,5 \%)$ versus 5 von 14 ( $=36 \%$ ) verloren, in der Gewichtsklasse IV (1251 bis $1500 \mathrm{~g})$ überlebten alle 40 gegenüber einer Verlustrate von 3 aus 14 $(=21 \%)$ im vorausgehenden Zeitraum.

Bezogen auf die Schwangerschaftswochen wurde die gleiche Tendenz deutlich (Abb. 2). Der Vergleich ist in der 24. und 25. SSW noch unmöglich, da diese extremen Frühgeburten vor 1984 nicht intensiv-medizinisch versorgt bzw. als Spätaborte behandelt wurden. Der Anteil dieser Kinder an der Gesamtmortalität unserer Klinik, schicksalsbestimmende Fehlbildungen ausgenommen, sank von $30 \%$ in den Jahren 1982/83 auf $18 \%$ im Zeitraum 1984/87. Gleichzeitig verdoppelte sich ihr Anteil an der gesamten Kinderzahl von 1 auf $2,4 \%$.

In Abbildung 3 und 4 ist die Frequenz der schweren Hirnblutungen sowie des schweren Atemnotsyndroms dargestellt. Der Vergleich der Jahrgänge zeigt einen signifikanten und deutlichen Rückgang der Inzidenz schwerer Hirnblutungen (Grad III und IV). 1982/83 erlitten 13 von 45 Frühgeborenen eine dritt- oder viertgradige Blutung, demgegenüber waren es 1984/87 13 von 141 Kindern ( $p \ll 0,01$ ). Bemerkenswert ist, daß bei dieser Abnahme der Häufigkeit schwerer Blutungen kein Anstieg der Zahl leichter Blutungen (Grad I und II) beobachtet wurde (22,2 versus $24,8 \%$ ).

Auch eine Tendenz zur Verminderung des schweren Atemnotsyndroms war erkennbar: 1982/83 zeigten 20 von 45 Frühgeborenen ein schweres ANS Grad III oder IV. 1984 bis 1987 waren 43 von 141 Kindern betroffen. Diese tendenzielle Veränderung erreicht jedoch keine statistische Signifikanz. 
geführte Untersuchung eine deutliche Verbesserung der Mortalitätsziffern in diesem perinatologischen Zentrum im Vergleich zur Umgebung nachwies (20). Dieses Ergebnis wurde auch in einer deutschen Regionalanalyse gefunden $(16,23)$. Trotz gut organisierter Abhol- und Flugdienste für Frühgeborene unterschieden sich die Behandlungsergebnisse von „in born"- und „out born"-Fällen deutlich. Bei konsequenter Regionalisierung der Risikogeburtshilfe wie in Skandinavien und in den USA wurde eine niedrige Neugeborenen-Sterblichkeit erreicht $(1,15)$. Alle diese Untersuchungen weisen deutlich den „Uterus als bestes Transportmedium“ aus. Derartige Zentralisierungszentren sind in der Bundesrepublik Deutschland im Ansatz vorhanden und werden im Einzugsgebiet des Perinatologischen Zentrums Erlangen zunehmend sichtbar. Das Risikokollektiv der kleinen Frühgeborenen betrug vor wenigen Jahren bei uns wie anderorts (12) konstant weniger als $1 \%$ aller Schwangerschaften. 1987 stieg der Anteil der kleinen Frühgeborenen in Erlangen auf 2,4\%, im ersten Halbjahr 1988 hat sich der Anteil bereits auf $4.1 \%$ erhöht.

Mit der Entwicklung einer engen Verflechtung zwischen Geburtshilfe und Pädiatrie wird die Frage der geburtshilflichen Entscheidungen zunehmend von neonatalen Gesichtspunkten beeinflußt. Eine der offenen Fragen zielt auf den Stellenwert des Entbindungsmodus.

Zur Geburtsleitung in der Gewichtsgruppe 500 bis $1500 \mathrm{~g}$ sind in den letzten Jahren zahlreiche Literaturmitteilungen $(2,7,9,25)$ erfolgt, ohne daß allgemein gültige Richtlinien erstellt werden konnten. Obwohl vieles für die Sectio als den weniger traumatisierenden Entbindungsmodus spricht, wird die generelle Entbindung durch Kaiserschnitt nicht empfohlen $(9,25)$. Erste Hinweise auf mögliche Vorteile der elektiven Schnittentbindung ergab eine vergleichende Studie von Kindern mit einem Geburtsgewicht von 1000 bis $1500 \mathrm{~g}$ aus Kalifornien. Eine dreifach höhere Sectiofrequenz führte zu einer Halbierung der perinatalen Mortalität (24). Zum gleichen Ergebnis kam eine retrospektive Studie aus dem University College Hospital London in der Gewichtsgruppe von 500 bis $1000 \mathrm{~g}$, gültig sowohl für Schädellagen wie für BEL (13). Bei höherem Geburtsgewicht (1001 bis $1500 \mathrm{~g}$ ) war der Unterschied nur noch für die BEL-Geburten signifikant. Auch in der hier vorliegenden Untersuchung war eine ab 1984 fast dreifach höhere Sectiorate mit einer Senkung der Mortalität auf die Hälfte verbunden. Trotzdem kann keine generelle Aussage zugunsten der obligaten Schnittentbindung gemacht werden, Der alleinige Bezug auf die Mortalität und Morbidität der Kinder berücksichtigt nicht die jeweilige Ausgangssituation und die Indikationsstellung zur Sectio, die unabhängig von der Kindslage auf sehr verschiedenen perinatalen Umständen beruhen kann. Eine Analyse nach der unterschiedlichen Ausgangssituation steht noch aus, erfordert allerdings größere Fallzahlen. Es ist jedoch bemerkenswert, daß Sectiokinder auch gegenüber vaginalen Schädellagen-Geburten deutlich besser abschnitten. Dies war besonders eindrucksvoll bei Berücksichtigung der schweren Hirnblutung als wichtigstem Morbiditätskriterium dieser kleinen Kinder und gilt für beide untersuchten Gewichtsklassen sowohl für die BEL wie auch für die Schädellage, wenn auch in unterschiedlicher Frequenz.

Die Entwicklung eines schweren Atemnotsyndroms war dagegen relativ unabhängig vom Entbindungsmodus und konnte durch die Sectio kaum gesenkt werden. Dieser Befund entspricht der Erwartung, wird das ANS doch in erster
Linie durch den Grad der Lungenentwicklung bei der Geburt bestimmt (3). Dagegen wurde aus der Untersuchung deutlich, daB sich die Kombination Hirnblutung und schweres ANS besonders ungünstig auswirkt. Der Vermeidung des Geburtstraumas kommt bei ANS-gefährdeten Kindern daher die allergrößte Bedeutung zu. Für BEL-Kinder erscheint die Sectio obligat, für Schädellagen-Kinder muß die jeweilige geburtshilfliche Situation berücksichtigt werden. Eine vaginale Schädellagen-Entbindung kann schonend verlaufen, wenn die geburtshilflichen Bedingungen günstig sind. Diese Bedingungen müssen jedoch noch herausgearbeitet werden und bedürfen weiterer Untersuchungen.

Nach vorzeitigem Blasensprung und Entwicklung eines Amnion-Infektionssyndroms verschlechtert sich die Ausgangssituation für Frühgeborene erheblich (11). Dabei besteht eine enge Beziehung zwischen der Latenzzeit des vorzeitigen Blasensprungs und der Entbindung. Bei 13 der 16 kindlichen Sepsisfälle aus der eigenen Untersuchung lag der Blasensprung mehr als 48 Stunden zurück. 8 Kinder starben ohne Nachweis einer schweren Hirnblutung, also infolge der perinatalen Infektion. Diese erschreckenden Befunde müssen Anlaß zum Umdenken sein. Nach einer Expertenumfrage von 1977 wurde überwiegend vor der 35. SSW oder unterhalb eines Körpergewichtes von 2000 g eine zunächst abwartende Geburtsleitung nach vorzeitigem Blasensprung empfohlen, um die Lungenreifungsprophylaxe durchführen zu können (19). Hier waren die Ansichten zwar nicht einheitlich, es überwogen aber die Stimmen für ein exspektatives Vorgehen. Die damals gegebene Empfehlung, die Geburt unter Einsatz der Tokolyse und gleichzeitiger Induktion der Lungenreifung mit Glukokortikoiden so lange hinauszuzögern, bis die kindliche Lungenreife erreicht ist, gilt weitgehend auch heute noch (8). Dies entsprach auch dem Vorgehen an der Erlanger Universitäts-Frauenklinik bis 1984. Ab diesem Zeitpunkt wurde nach kürzeren Latenzzeiten häufiger aktiv entbunden. Bei Hinweis auf ein beginnendes Amnion-Infektionssyndrom wurde ohne Kortikoid-Prophylaxe auch bei einem Schätzgewicht von weniger als $1000 \mathrm{~g}$ unter hochdosierter Antibiotika-Gabe sofort entbunden. In den letzten 2 Jahren wurde hierfür dem Anstieg des C-reaktiven Proteins (CRP) großes Gewicht beigemessen. Nach AbschluB der ANS-Prophylaxe (48 Stunden) wurde auch ohne Zeichen einer Infektion häufiger entbunden. Die Tokolyse wurde bei zunehmender Wehentätigkeit nach Ablauf der 48Stundenfrist nur noch zurückhaltend indiziert. Intravenöse Höchstdosen mit erhöhtem mütterlichen Risiko wurden vermieden. Bei unreifer Zervix und Schädellage oder bei Beckenendlage des Kindes wurde die Sectio vorgenommen. Als untere Gewichtsgrenze wurde ein Schätzgewicht von $750 \mathrm{~g}$ festgelegt. Dieses Vorgehen bewirkte vornehmlich die ab $1984 \mathrm{zu}$ verzeichnende Steigerung der Sectiofrequenz. Während die Latenzzeit von mehr als 48 Stunden zwischen vorzeitigem Blasensprung und Geburt von 38 auf $20 \%$ abnahm, sank die Sepsisfrequenz nahezu auf die Hälfte ( $39 \%$ versus $21 \%$ ). Diese Verkürzung der Latenzzeit führte nicht zu einer Zunahme der durch die Unreife bedingten Komplikationen, sondern mit dem Rückgang der Hirnblutungen, des Atemnotsyndroms und der perinatalen Infektionen zur Senkung der Morbidität und Mortalität.

Ein häufiges Problem bei der Behandlung der frühen Frühgeburt ist die Unsicherheit in der Einschätzung des erreichten Gestationsalters. Dies führt nicht selten zu einer oft fatalen Fehleinschätzung der Prognose kleinster Frühgebore- 
ner. Die Unterschätzung des Gestationsalters kann bei Fehleinschätzung der Überlebenschancen zu falschem, abwartenden Verhalten führen, wenn etwa die Sectio als die geeignete geburtshilfliche Maßnahme indiziert wäre. Aus unseren eigenen Zahlen läßt sich deutlich entnehmen, daß die Berücksichtigung des Gestationsalters zu weitaus größerer Ungenauigkeit in der Überlebensprognose als die ultrasonographische Gewichtsschätzung führt. Erst der vorrangige Bezug auf das Schätzgewicht führte zu einer besseren Entscheidungshilfe für das geburtshilfliche Vorgehen. Das mit Hilfe der dreiparametrischen Schätztabellen nach Hansmann ermittelte, zu erwartende Geburtsgewicht erwies sich auch in der Hand eines groBen geburtshilflichen Teams als sehr zuverlässig. Auch andere Arbeitsgruppen kamen zu ähnlichen Ergebnissen: So erwies sich die antepartale Schätzung des Kindsgewichtes in den Ergebnissen der University of Southern California, Los Angeles, aus den Jahren 1975 bis 1977 als schicksalsbestimmend. Hier wurde bei mehr als $1000 \mathrm{~g}$ Schätzgewicht aktiv überwacht und häufig durch Sectio caesarea entbunden. Bei den weniger als $1000 \mathrm{~g}$ Geschätzten verhielt man sich passiv. Im Gewichtsgruppenvergleich zeigten die Kinder mit einem tatsächlichen Gewicht über $1000 \mathrm{~g}$ bei korrekter Voraussage eine deutlich bessere Entwicklung als Kinder mit gleichem Geburtsgewicht, aber - falsch - zu niedriger Schätzung (18). Aufgrund der gewichtsbezogenen Analyse der Erlanger neonatalen Ergebnisse kann ab einem geschätzten Geburtsgewicht von $1000 \mathrm{~g}$ ein aktives geburtshilfliches Vorgehen als sicher gerechtfertigt angesehen werden. In der Gewichtsklasse zwischen 750 und $1000 \mathrm{~g}$ ermutigen Einzelverläufe ebenfalls zu aktivem Vorgehen, so daß wir derzeit die mit einem gesunden Überleben zu vereinbarende Grenze bei $750 \mathrm{~g}$ ziehen. Das kleinste gesund überlebende Frühgeborene wog im Untersuchungszeitraum $540 \mathrm{~g}$.

Das Atemnotsyndrom gilt nach wie vor als die häufigste neonatale Todesursache von Kindern unter $2500 \mathrm{~g}$ Geburtsgewicht (16). Zudem stellt es einen wesentlichen Risikofaktor für die Entstehung einer Hirnblutung dar (4). Dementsprechend fanden wir in der eigenen Untersuchung eine statistisch hohe Koinzidenzrate von Hirnblutung und Atemnotsyndrom. Da die Kortikoid-Prophylaxe in den hier verglichenen Zeiträumen gleich häufig vorgenommen wurde, kann ihr im zeitlichen Vergleich kein wesentlicher Beitrag zur beobachteten Senkung der Häufigkeit des schweren ANS und der Mortalität zuerkannt werden. Ihre Wirksamkeit in der ANS-Prophylaxe konnte nur in der Gewichtsgruppe 500 bis $1000 \mathrm{~g}$ statistisch signifikant nachgewiesen werden. Bei Kindern mit einem Geburtsgewicht von 1001 bis $1500 \mathrm{~g}$ fand sich dagegen keine nachweisbare Wirkung der ANS-Prophylaxe. Eine Antibiotika-Gabe während der Kortikoid-Prophylaxe, wie von einigen Autoren empfohlen (8), wurde nicht vorgenommen. Die Amnion-Infektionsrate war in beiden Gruppen mit und ohne Kortikoid-Applikation gleich groß (13\% versus $11 \%$ ), so daß auch aus dieser Untersuchung keine Indikation zur generellen Antibiotika-Prophylaxe abgeleitet werden kann.

Ein aktiveres geburtshilfliches Vorgehen zur Vermeidung traumatisierender Entbindung und Verminderung damit verbundener schwerer Hirnblutungen bei kleinsten Frühgeborenen und gleichzeitiger Senkung der Infektionsrate kann nur vertreten werden, wenn eine Erstversorgung durch ein geschultes pädiatrisches Team möglich ist. Prospektives geburtshilfliches Denken im Hinblick auf Planung und Organisation der Frühgeburt ist dabei von entscheidender
Bedeutung für ein gutes Ergebnis. Die in unserem zweiten Untersuchungszeitraum beobachtete fast ausnahmslose Anwesenheit des Pädiaters bedeutete, daß der Geburtshelfer nicht von der Frühgeburt überrascht wurde, sondern rechtzeitig gehandelt hat. Nur so ist auch die hervorragende Korrelation zwischen guter kindlicher Prognose und Präsenz des Pädiaters bei der Geburt zu deuten. Es versteht sich von selbst, daß jede auch nur kleinste Lücke in der postnatalen Versorgung, die aktiv betrieben werden muß, zu deletären Folgen für das Kind führt (23). Die räumliche Nähe der geburtshilflichen Abteilung zur Kinderklinik ist dabei wichtig. Ist keine neonatologische Intensivstation mit der Frauenklinik direkt verbunden, sollte der erste und vielleicht wichtigste Schritt zur prospektiven Organisation der Frühgeburt der „Transport in utero" sein. Dies sollte dabei so rechtzeitig geschehen, daß eine pathologische Situation nicht verschleppt wird. Eine späte Verlegung führte in unserer Untersuchung bei einigen Fällen zu unterbrochenem Monitoring und Situationen, die keine wirksame Einflußnahme und Leitung der Geburt zuließen. Die Ergebnisse waren in diesen Fällen schlecht.

Daß diese Erkenntnisse immer mehr Beachtung finden, ist am nachweisbaren Effekt der Regionalisierung im Einzugsbereich des Perinatalzentrums Erlangen meßbar geworden.

\section{Literatur}

1 Anderson, C. L., S. Aladjem, O. Ayuste, C. Caldwell, M. Ismail: An analysis of maternal transport within a suburban metropolitan region. Am. J. Obstet. Gynecol. 140 (1981). 499.

2 Duenholter, J. H., C. E. Wells, J. S. Reisch, R. Santos-Ramos, J. H. Jimenez: A paired controlled study of vaginal and abdominal delivery of the low birthweight breech fetus. Obstet. Gynec. 54 (1979). 310 .

3 Ewerbeck, H., P. Groneck: Das extrem unreife Frühgeborene - ein Dilemma für Geburtshelfer und Pädiater. Geburtsh. Frauenheilk. 43 (1983). 99.

4 Garcia-Prats, J. A., R. S. Procianoy, J. M. Adams, R. J. Rudolph: The hyaline membrane disease - intraventricular hemorrhage relationship in the very low birthweight infant: perinatal aspects. Acta Paed. Scand. 71 (1982), 79.

5 Giedion, A., H. Haefliger, P. Dangel: Acute pulmonary X-ray changes in hyaline membrane disease treated with arteficial ventilation and positive end-expiratory pressure (PEP). Pediatr. Radiol. 1 (1973). 145-152.

6 Hansmann, M., H. Schuhmacher. H. Voigt: Mehrparametrische nichtlineare Gewichtsschätzung mittels Ultraschall unter Berücksichtigung des Gestationsalters. In: Kratochvil, Reinold: Ultraschalldiagnostik. Georg Thieme, Stuttgart 1978.

7 Hickl, E. J.: Zur Frage der Optimierung der geburtshillich-pädiatrischen Versorgung von Frühgeborenen. Gynäkologe 20 (1987). $41-47$.

* Hochuli, E.: Geburtshilfe, Gynäkologie und Grenzgebiete, Hans Huber Verlag, Bern - Stuttgart-Toronto 1985.

9 Hochuli, E.: Geburtsleitung bei früher Geburt. Gynäkologe 20 (1987), 32-40.

10 Hohenauer. L.: Intrauterine Wachstumskurven für den Deutschen Sprachraum. Z. Geburtsh. u. Perinat. 184 (1980). 167.

11 Kitchen. W., G. W. Ford, C. W. Doyle. A. L. Rickards, J. V. Lissenden, J. R. Pepperell. J. E. Duke: Cesarean section or vaginal delivery at 24 to 28 weeks gestation: comparison of survival and neonatal and two-year morbidity. Obstet. Gynec. 66 (1985). 149.

12 Kubli. F: Das Risiko-Neugeborene im Spannungsfeld zwischen Geburtshilfe und Kinderheilkunde. Referat auf der Tagung der Deutschen Gesellschaft für Gynäkologie und Geburtshilfe. Düsseldorl. September 1986

13 Liu, D. T. Y., D. V. J. Fairweather: The management of preterm labour. In: Preterm Labour, Butterworths. Int. Med. Rev. Obstet. Ed. Elder Hendricks (1981), 231. 
14 Mentzel, M.: Das sehr unreife Frühgeborene. Gynäkologe 20 (1987), 48 .

15 Modanlou, H. D., W. Dorchester, P. K. Freemann, C. Rammet: Perinatal transport to a regional center in a metropolitan area: Maternal versus neonatal transport. Am. J. Obstet. Gynecol. 138 (1980), 1157.

16 Obladen, M.. U. Heemann, K. M. Hennecke, I. Hanssler: Ursachen neonataler Letalität 1981-1983: Eine regionale Analyse. Z. Geburtsh. u. Perinat. 189(1985), 181-187.

17 Papile, L. A.. J. Burstein, R. Burstein. H. Kuffler: Incidence and evalution of subependymal and intraventricular hemorrhage: a study of infants with birth weight less than $1500 \mathrm{~g}$. J. Pediatr. 92 (1978). $529-534$.

is Paul, R. H., K. S. Koh, A. H. Monfared: Obstetric factors influencing outcome in infants weighing from 1001 to 1500 grams. Am. I. Obstet. Gynecol. 133 (1979). 503.

19 Plotz, E. J.. K. Schander: Der vorzeitige Blasensprung (Ein schriftliches Symposium). Geburtsh. und Frauenheilk. 37 (1977). 997.

20 Reynolds. E. O. R.. A. C. Stewart: Intensive care and the very low birthweight infant. Lancet II (1979), 254.

21 Riegel, K.: Säuglingssterblichkeit - Verlauf. Ursachen, Verbesserungsmöglichkeiten. Monatsschr. Kinderheilk, 131 (1983), 552.

22 Stewart, A.: Follow up bei Kindern mit sehr niedrigem Geburtsgewicht (VL.BW). In: Huch. A. R. Huch, G. Duc, G. Rooth (Hrsg.): Klinisches Management des „Kleinen“ Frühgeborenen (<1500 g). Thieme Verlag. Stuttgart-New York 1982.

${ }^{23}$ Segerer. H., W. Landendörfer. K. H. Deeg. K. Richter: Reduktion von Hirnblutungen und Atemnotsyndrom bei Frühgeborenen durch Vermeidung einer perinatalen Asphyxie. Monatsschr. Kinderheilk. $136(1988), 1$.

${ }^{24}$ Williams, R. L., P. M. Chen: Identifying the sources of the recent decline in perinatal mortality rates in California. N. Engl. J. Med. 306 (1982), 207.

25 Wulf, K. H.. E. Kastendieck. D. Seelbach-Göbel: Zum Geburtsmodus bei Frühgeborenen - abdominal oder vaginal? Z. Geburtsh. Perinatol. 188 (1984). 249-255.
OA Dr. med. H. J. Voigt

Klinik für Frauenheilkunde mit Poliklinik und Hebammenschule der Universität Erlangen-Nürnberg

Universitätsstr. 21-23

8520 Erlangen 